\title{
Microvascular Abnormalities in Depression
}

\author{
Evgenii Sadykov, ${ }^{1}$ Ladislav Hosak,,${ }^{1,2,}$ Jan Studnicka, ${ }^{3}$ Xavier Fung, ${ }^{4}$ Khurum Hakeem, ${ }^{4}$ Ashwin
}

\author{
Mangarai, ${ }^{4}$ Ishleen Kaur, ${ }^{4}$ Gloria Mbuebue, ${ }^{4}$ Stefanos Kateroglou, ${ }^{4}$ and Mikela-Rafaella Siligardou ${ }^{4}$ \\ ${ }^{1}$ Department of Psychiatry, Charles University in Prague, School of Medicine in Hradec Kralove, Czech Republic \\ ${ }^{2}$ Department of Psychiatry, University Hospital, Hradec Kralove, Czech Republic \\ ${ }^{3}$ Department of Ophthalmology, Charles University in Prague, School of Medicine in Hradec Kralove, and University Hospital Hradec Kralove, Czech Republic \\ ${ }^{4}$ Charles University in Prague, School of Medicine in Hradec Kralove, Czech Republic \\ "Corresponding author: Ladislav Hosak, Dpt. of Psychiatry, University Hospital, Sokolska 581, 500 05 Hradec Kralove, Czech Republic. Tel: +420-495832228, Fax: +420-495833041,
} E-mail: hosak@lfhk.cuni.cz

Received 2016 January 24; Revised 2016 June 20; Accepted 2016 December 09.

\begin{abstract}
Context: Depression is a risk factor for vascular disease and vice versa. Identification of biological mechanisms common for depression and vascular pathology at microvascular level, and understanding their clinical significance may contribute to better treatment and prognosis of this combined physical/psychiatric disorder.

Evidence Acquisition: We searched the PubMed computer database for the following key words "Microvascular AND Depression" on the 28th of October, 2015 to obtain relevant articles, which were consequently summarized to facilitate further research in the field of psychiatry.

Results: The majority of studies support the suggestion that microvascular abnormality is associated with depression even if several documents do not necessarily support this idea. It seems probable that microvascular abnormality is only relevant in particular subtypes of depression, for example depression due to a general medical condition, late-life depression, or pharmacoresistant depression. The biological mechanisms by which microvascular abnormality is interconnected with depression may cover genetics, epigenetics, endothelial dysfunction, inflammation or hyperhomocysteinemia.

Conclusions: Further research should be aimed at identifying biological mechanisms common in depression and microvascular pathology, including genetics and epigenetics, longitudinal studies of this subcategory of patients with depression, and possible pharmacotherapy overcoming the usual non-responsiveness. An active detection and aggressive treatment of physical diseases associated with microvascular pathology and depression, like hypertension, diabetes or inflammation, is warranted. This way, the patients experience less distress, their quality of life will increase, and societal cost of this vascular/psychiatric disorder will be reduced.
\end{abstract}

Keywords: Depression, Microvascular Abnormalities, Neuroimaging, Retina, Biomarkers, Inflammation, Diabetes Mellitus, Hypertension

\section{Context}

Depression is a common illness worldwide, and about 350 million people are affected. Females are more susceptible to depression and anxiety in comparison to males. Especially in long-term case and moderate to severe situations, depression can become a serious health condition. Patients with depression experience great distress and function poorly at work, at school and in their family. At its worst, depression may lead to suicide. Every year, an estimated 800000 people die from suicide, globally, the majority being males $(1,2)$. Mental disorders also have serious economic consequences: depression was estimated to cost at least 800 billion USD during 2010 in loss economic output, a sum expected to more than double by 2030 (3). If the disease burden was measured using disabilityadjusted life-years (DALYs), depression accounted for $10 \%$ of all DALYs in individuals aged 15 to 44 years in 1995 . Fur- thermore, this figure is expected to rise to $15 \%$ by the year 2020 , rendering depression second in terms of worldwide diseases burden, with only ischemic heart disease more widespread (4).

Due to the fact that depression increases not only the rate of vascular morbidity but also the mortality rate associated therein, we can view depression as one of the risk factors for vascular disease. Community-based studies have shown that there is an association between depression and heightened rates of cardiovascular disease mortality in adults and the elderly. Depression is also an independent risk factor for heart failure and death after myocardial infarction (5). An early treatment of depression may stop this pathological process. On the other hand, depression may be triggered by a vascular disease. The prevalence of depression-related symptoms during a post stroke time period of 3- to 6-months lies between $29 \%$ and 36\%. Af- 
ter the initial stroke,20\% of patients exhibit symptoms of depression more than 2 years (6).

Examination of the retinal vessels, in addition to brain imaging techniques, could indicate the vessel condition of the brain. The main vessel of the eye is the artery of the central retina, which is a branch of the ophthalmic artery, with the ophthalmic artery itself being a branch of the internal carotid artery. This artery of the central retina travels inferior to the optic nerve within its dural sheath directly to the eyeball and branches out along the retina's internal surface. These terminal branches are the sole supply of blood to the larger part of the retina. As vertebrate embryos develop, the retina and optic nerve are outgrowths of the brain, thus making the retina part of the central nervous system(CNS). It is in fact brain tissue and the only nonsurgically observable part of the CNS (7). In addition, the examination of retinal vessels is relatively easy, available, quick and cheap, and applicable in living subjects (8).

\section{Objectives}

The aim of this article was to review the literature relevant for microvascular abnormalities in depression in order to facilitate further research in this field of psychiatry.

\section{Evidence Acquisition}

Firstly, the PubMed computer database was searched on the 28th of October, 2015 using the following key words "microvascular and depression". Next, the content of relevant articles was summarized. Regarding the inclusion criteria, we preferred publications related to humans compared to studies on research animals, and articles covering living subjects to postmortem studies. We used original research articles as well as reviews. Exclusion criteria included animal studies, postmortem studies unrelated to microvascular abnormalities and articles discovered only by accident, unrelated to the topic of our review. Because our manuscript is only a simple review including a broad spectrum of studies with different research methods, we did not apply statistical analysis of our results.

\section{Results}

\subsection{Postmortem Studies}

Santos et al. (2009) investigated 41 post-stroke patients, who underwent autopsy, after excluding patients with neurological disease such as Alzheimer's disease or Parkinson's disease. Among 20 post-stroke depression
(PSD) cases, 7 were untreated and 8 were treated with Selective Serotonin Re-uptake Inhibitor (SSRI) or Tricyclic Antidepressants (TCA). In 5 PSD cases, data on antidepressant treatment were incomplete for the entire post-stroke survival period. Postmortem tissues were examined investigated for signs of macroscopic vascular lesions, for example cerebral infarction, lacunes, and cerebral hemorrhage. The results showed that small vascular lesions were associated with PSD's occurrence. This was the case for thalamic and basal ganglia lacunes bilaterally. It was also true for deep white matter lacunes located in the right hemisphere, which were found to occur significantly more often in PSD cases. The combined lacune score (thalamic plus basal ganglia plus deep white matter) accounted for $25 \%$ of the variability in terms of the occurrence of PSD (9).

Santos et al. (2010) undertook an in-depth postmortem analysis of small macrovascular and microvascular pathology in the brains of 38 patients aged 65 to 89 (16 men and 22 women) with late-onset major depression(LOD) and 29 (age- and gender-matched) controls without depression. The analysis showed that the occurrence of LOD was not associated with the presence of microvascular ischemic lesions nor with the existence of lacunes (10).

Ballard et al. (2000) investigated 30 participants suffering from cerebrovascular disease and significant cognitive impairment (VaD), with special focus given to patients with small infarct volumes (less than $15 \mathrm{~mL}$ ). The study included postmortem histological analysis. Thirty patients with VaD had a mean volume of infarction of $24.4 \mathrm{~mL}$. The results showed that patients with VaD were significantly more likely to have major depression (27 vs 5\%; OR 6.4; 95\% CI 1.2 - 32.8) than control patients with Alzheimer's disease. Within the VaD patients, depression was associated with an infarction volume of $<15 \mathrm{~mL}$ and areas of microinfarction (11).

Thomas et al. (2001) investigated postmortem tissues to ascertain whether depression developing late in life correlated with atheromatous changes in vessels (medium and large) and cerebral microvascular disease. Postmortem tissue was collected from 20 patients who had had a history of one or more episodes of major depression as based on the diagnostic and statistical manual of mental disorders, 4th Edition (DSM-IV) major depression and 20 control subjects. A significant increase in atheromatous disease was present in the group with depression $(\mathrm{P}=0.023)$. However, there were no differences observed for microvascular disease, neither in the brain generally or in the frontal lobes specifically. The authors suggested that screening for atheromatous disease should be available for those with depression later in life, the reason being twofold: firstly, the disease functions as a factor adding to 
the depression itself; secondly, the condition predisposes depression sufferers to other vascular disorders (12).

Sinka et al. (2012) studied and analyzed the postmortem morphological parameters of capillaries in the area of the anterior cingulate cortex (ACC), which could affect brain perfusion in schizophrenia $(\mathrm{N}=8)$, sporadic bipolar disorder $(\mathrm{N}=10)$, sporadic major depression $(\mathrm{N}=$ $8)$, and age and gender matched control cases $(\mathrm{N}=7)$. The results showed that the mean capillary diameter significantly decreased in bipolar and unipolar depression cases whereas schizophrenia patients showed similar results to the controls (13).

\subsection{Neuroimaging}

Hajjar et al. (2011) investigated the association between hypertension, white matter hyperintensities and concurrent impairments in mobility, cognition and mood of 4700 adults aged 65 years and older. The authors discovered that there were concurrent impairments present in 498 (11\%) of the subjects, and in 3086 (66\%) all three measures were found intact. Hypertensive individuals were more likely to be impaired at baseline (OR 1.23; 95\% CI 1.04 - 1.42; $\mathrm{P}=0.01$ ) and become impaired during the follow-up (HR $=1.3 ; 95 \%$ CI $1.02-1.66 ; \mathrm{P}=0.037)$. There was an association between impairments in three domains $(\mathrm{P}=0.007)$ and a greater degree of white matter hyperintensities, thus mediating the association with hypertension. The authors concluded that the risk of concurrent impaired mobility, cognition, and mood increase with the presence of hypertension, with microvascular brain injury partly mediating this association (14).

Wolfson et al. (2013) measured brain white matter hyperintensities (WMH), blood pressure, mobility, cognition and depression (center for epidemiologic studies depression scale) of 67 participants aged 75 to 89 during 4 years. The results showed that WMH increased from $0.99 \%$ of intracranial cavity volume at baseline to $1.74 \%$ on average, after 4 years. Regression analyses showed that three of five mobility measures, two of four cognitive measures, as well as depression itself, were associated with the baseline WMH and 4-year WMH $(\mathrm{P}=0.005)(15)$.

Patankar et al. (2007) hypothesized that virchow-robin spaces (VRS) dilatation may be a predictor of antidepressant monotherapy resistance in elderly patients suffering from depression. Virchow-Robin Spaces are spaces in cerebrospinal fluid and are associated with microangiopathy of small cerebral vessels. Fifty sufferers of Late onset (age over 60 years) major depressive disorder (29 responders to monotherapy, 21 nonresponders to monotherapy) and 35 normal volunteers were recruited. The authors assessed deep white matter lesions (WML) and periventricular hyperintensities (PVH) using the Scheltens rating scale score. The results showed that in the monotherapy resistant group there was a trend for greater WML Scheltens scores compared to responders and control subjects. It was only when the basal ganglia VRS score was used that a statistically significant difference appear $(\mathrm{P}<0.05)$. The basal ganglia VRS score accounted for $38 \%$ of the variance of nonresponse in the multiple regression model, and the Scheltens PVH score produced the additional $6 \%$ of the variance (16).

\subsection{Myocardial Imaging}

Vaccarino et al. (2009) used positron emission tomography to find the association between major depression and coronary flow reserve for 289 male middle-aged twins, including 106 twins (53 twin pairs) discordant for a lifetime history of major depressive disorder (MDD) (25 dizygotic and 28 monozygotic), and 183 control twins pairs free of MDD (76 dizygotic and 107 monozygotic). The results showed that no difference was found between twins with and without MDD in terms of myocardial ischemia. Among the dizygotic twin pairs who were discordant for MDD, the coronary flow reserve (CFR) was $14 \%$ lower in MDD twins than in their non-MDD brothers (2.36 vs $2.74 ; \mathrm{P}=0.03$ ), and also significantly lower than in the control twins. Those pairs, who were monozygotic discordant, lacked this association. The results yield important new information which points at microvascular disease as one of the key component of the shared pathway between MDD and coronary heart disease (17).

\subsection{Retinal Imaging}

Cheung et al. (2009) investigated 10364 Caucasian and African Americans aged 48 to 73 years to establish a possible association between microvascular changes in the retina with vital exhaustion (including depressive symptoms). Vital exhaustion was modestly associated when retinopathy was present (OR 1.27; 95\% CI 1.01-1.59), particularly retinal hemorrhages and generalized retinal venular widening (18).

Jensen et al. (2009) investigated retinal photographs with retinal microvascular signs and psychosocial risk factors of 6814 adults aged 45 to 84 years. The authors discovered that subjects who did not appear to have access to emotional support (enriched social support instrument score) had a $60 \%$ greater chance of retinopathy than subjects with this support (OR 1.6; 95\% CI 1.3 - 2.0). Subjects exhibiting high scores on the Spielberger trait anxiety scale and subjects with high depressive symptoms (center for epidemiologic studies depression) were more likely to suffer from retinopathy (OR 1.4; 95\% CI 1.1 - 1.9; and OR 1.5; 95\% CI 1.2 - 1.9, respectively). In this cross-sectional study, the 
absence of emotional support, increased trait anxiety, and more depressive symptoms were associated with signs of retinopathy, independently of the other known risk factors (19).

Ikram et al. (2010) tested whether small retinal arteriolar or larger venular calibers were associated with incidental late-life depression. With a total number of 3605 participants, those with incidental depression symptoms, found during follow up visits and monitoring, were identified. After a mean follow up of 9 years, 555 patients (15.4\%) developed late-life depression. There was no correlation between retinal vascular calibers and incidental late life depression, neither in small arteriolar or larger venular calibers (20).

Sun et al. (2007) performed a large population-based cohort study in order to examine the association between the manifestations of depression and microvascular abnormalities of retina like retinopathy, arteriovenous nicking, generalized arteriolar narrowing in the retina, focal arteriolar narrowing and generalized retinal venular dilatation in 2420 subjects. Data provided from digital retinal photographs, as well as the short-item centers for epidemiologic studies depression scale (CES-D), were analyzed. In conclusion, no association was found between retinal microvascular abnormalities and symptoms of depression (21).

Kim et al. (2011) performed an assessment of retinal microvascular signs and functional loss of 1744 participants (mean age 78) free of stroke. The results showed that retinal signs (generalized arteriolar narrowing) had correlations with depressive mood. The authors suggested that retinal signs might represent microvascular damage due to various vascular insults including hypertension, inflammation, smoking, and diabetes (22).

Li et al. (2013) measured symptoms of antenatal depression, anxiety, and sleep quality as well as retinal vascular caliber of 952 Asian pregnant females aged 18 to 46 years. They applied retinal photography and assessments of the edinburgh postnatal depression scale (EPDS), statetrait anxiety inventory (STAI), and pittsburg sleeping quality index (PSQI). In multiple linear regression models, each standard deviation (SD) increase in the Edinburgh postnatal depression scale and the pittsburgh sleep quality index was significantly linked to an $0.80 \mu \mathrm{m}(\mathrm{P}=0.03)$ and $1.22 \mu \mathrm{m}(\mathrm{P}=0.01)$ expansion of the retinal arteriolar caliber, respectively. The authors concluded that study reveals the link between antenatal depressive symptoms and poor sleep and the widening of the retinal arteriolar in pregnant females (23).

Meier et al. (2014) conducted a research on the link between retinal vessel damage, anxiety and depression in young adults and adolescents. The tests have been con- ducted on 865 participants during years 2000 to 2013 . They included the somatic and psychological health report (SPHERE) questionnaire, which contains subscale scores related to depression, anxiety and overall mental health. For the measurement of retinal vessel caliber, stereoscopic optic disk-centered photographs were taken. The results were presented as central retinal arteriolar equivalent (CRAE) and central retinal venular equivalent (CRVE). The results showed that symptoms of depression and anxiety showed a positive association with a wider retinal arteriolar caliber $(\mathrm{P}=0.016)$, even after adjusting for other cardiovascular risk factors $(\mathrm{P}=0.025)$, but not with CRVE. This shows that pathological microvascular mechanisms associated with depression/anxiety may be operative from a young age (24).

Nguyen et al. (2010) conducted a clinical study to examine whether retinal vascular luminal size was an indicator of microvascular disease and depression in patients with type 2 diabetes. The study participants were people with type 2 diabetes as well as with major depression $(\mathrm{n}=$ 43), with type 2 diabetes without depression $(n=49)$, and subjects without diabetes or depression $(n=54)$. The participants with type 2 diabetes and depression had a bigger retinal vascular lumen compared with those without depression and healthy controls $(\mathrm{P}=0.002$ for arteriolar and $\mathrm{P}=0.02$ for venular caliber). This supports the suggestion that early microvascular disease in diabetes type 2 may contribute to depression (25).

Nguyen et al. (2008) evaluated the correlation between microvascular disease and depression through a crosssectional study performed on patients with diabetes mellitus type 2. For this reason, a sample of 99 participants was recruited, of whom 38 healthy, neither diabetic nor mood-affected participants represented the control group, 27 were diabetic patients without mood disorders, and 34 were type 2 diabetes patients with major depression. Digital retinal photographs were used as a means to measure changes in retinal vessels' caliber, which is considered to have a primary role, early in the process of microvascular compromise. The group of diabetic patients with depression was associated with the largest mean retinal caliber measurement; the value of retinal caliber of diabetics without depression stood in between that of the other two groups, while the healthy, control group demonstrated the smallest retinal caliber measurement, respectively $(\mathrm{P}=$ 0.008 for arteriolar and $\mathrm{P}=0.03$ for venular caliber). However, an opposite trend was evident when the measurements were further adjusted for additional vascular risk factors. According to the authors, the increasing size of retinal caliber is mainly attributable to poor management of diabetes, insufficient glycemic control, inferior physical functioning, and poor compliance to diet and physical ex- 
ercise regimens (26).

\subsection{Inflammatory Markers}

Tchalla et al. (2015) measured Interleukin-6 (IL-6), soluble Intercellular Adhesion Molecule-1 (sICAM-1), circulating vascular cell adhesion molecule-1 (sVCAM-1) and Creactive protein (CRP) levels in plasma of 680 communitydwelling individuals, aged 65 years and older. Cell adhesion molecules are important biomarkers in those inflammatory processes which involve activation or damage to the endothelium and cells such as platelets. The authors also assessed magnetic resonance imaging (MRI) volumes of cerebral white matter hyperintensities (WMH) in a subset of 25 participants. Depressive symptoms were evaluated using the revised center for epidemiological studies depression scale (CES-D scale) (CESD-R; scores of $<16$ indicates no or minimal depressive symptoms and $\geq 16$ indicates the presence of moderate or severe symptoms). The results showed that 179 (27\%) subjects had a CESD-R score $\geq 16$. The mean sVCAM- 1 concentration of $1176 \mathrm{ng} / \mathrm{mL}$ was found in the group with CESD-R Scores $<16$ and $1239 \mathrm{ng} / \mathrm{mL}$ in those with CESD-R Scores $\geq 16(\mathrm{P}=0.036)$. The CESD$\mathrm{R}$ score indicated a positive association with $\operatorname{sVCAM-1}(\mathrm{r}=$ $0.11 ; \mathrm{P}=0.004)$. In a subset of subjects, sVCAM-1 concentration showed a positive correlation with cerebral $\mathrm{WMH}$ volume $(P=0.018)$. The mean IL-6 plasma level was significantly higher in those with depressive symptoms ( $P$ $=0.046)$. The authors concluded that increased plasma levels of pro-inflammatory mediators might function as a biomarker for the presence of cerebral microvascular disease in elderly individuals with symptoms of depression who live in a community (27).

Gorska-Ciebiada et al. (2015) studied the serum levels of C-reactive protein, Interleukin-6 (IL-6) and tumor necrosis factor (TNF- $\alpha$ ) in 276 elderly diabetic patients. Among them were 57 patients with depressive syndrome only, 25 patients with depression and a mild cognitive impairment (MCI), 62 patients with MCI only, and 132 diabetic controls with no depression or MCI. To assess depressive mood, the long version of the geriatric depression scale (GDS-30) was applied. In order to evaluate cognitive impairment, the Montreal Cognitive Assessment (MoCA) was used. In all groups of patients (depression, MCI or both), CRP, IL- 6 and TNF- $\alpha$ levels were significantly higher as compared to controls $(\mathrm{P}<0.001)$. The group with depressive mood and coexisting MCI displayed the highest level of inflammatory markers. Correlations were found among all the inflammatory markers in the group with patients exhibiting depressive mood only and in the group of subjects displaying depressive symptoms and coexisting MCI. There was a positive correlation in the GDS-30 score with levels of all inflammatory markers in the group with depressive mood without MCI, and with levels of CRP and TNF- $\alpha$ in the group with depressive mood and coexisting MCI. The authors showed that the presence of depressive syndrome is linked to higher levels of inflammatory markers in elderly patients suffering from diabetes. The presence of MCI in these depressed subjects has an additive effect on inflammatory mediator levels (28).

\section{Discussion}

Most of the studies support the suggestion that microvascular abnormality is associated with depression, whilst other works contradict this idea. It seems plausible that microvascular abnormality is only relevant in some subtypes of depression, for example, depression due to a general medical condition like atherosclerosis, myocardial ischemia, hypertension, diabetes mellitus or inflammation, late-life depression, or pharmacoresistant depression. For details and references, see Table 1.

Authors of several review articles tried to depict biological mechanisms by which microvascular abnormality is interconnected with depression (Figure 1). Camus et al. (2004) overviewed 37 articles. Among other genetic findings, a preliminary study revealed that the serotonin transporterl-linked polymorphism (5-HTTLPR) could increase the risk of geriatric depression. Literary, data suggest that the dual implication for serotonin in both depression and thrombogenesis supports the view that serotoninmediated platelet activation serves as a plausible pathophysiological process linking depression and vascular diseases. Apart from this, the allelic combination of two genes linked with an increase in the risk of myocardial infarction (angiotensin I converting enzyme and G-protein beta 3 subunit) was shown to heighten the vulnerability to depressive disorder (5). Camus et al. suggested three general potential pathways of association of vascular diseases and depression: 1. Direct influence of arteriosclerosis on the incidence of depression; 2. Direct impact of depression on the cardiovascular system; 3 . Either a shared common pathophysiological process or genetic background between depression and vascular disease.

According to Santos et al. (2009; 2012), depression may be induced either by the interrupting biogenic amine pathways through ischemic lesions in the brain, or the negative effect of proinflammatory cytokines released as a response to acute cerebral ischemia. There is also a positive relationship between the presence of the ApoE $\varepsilon 4$ allele and an increase in the risk of late-life depression. There exist three main etiopathogenetic pathways which have been implicated in both cardiovascular pathologies and latelife depression: hyperhomocysteinemia, endothelial dysfunction and inflammation. The authors also state 
Table 1. A General Overview of the Applied Studies

\begin{tabular}{|c|c|}
\hline Type of Studies & Authors and Year \\
\hline $\begin{array}{l}\text { Studies supporting the idea that microvascular abnormality is associated } \\
\text { with depression }\end{array}$ & $\begin{array}{l}\text { Ballard et al., 2000; Sinka et al., 2012; Nguyen et al., 2010; Hajjar et al., 2011; Kim et } \\
\text { al., 2011; Li et al., 2013; Wolfson et al., 2013; Meier et al., 2014; Tchalla et al., } 2015\end{array}$ \\
\hline $\begin{array}{l}\text { Studies contradicting the idea that microvascular abnormality is } \\
\text { associated with depression }\end{array}$ & Thomas et al., 2001; Santos et al., 2010; Sun et al., 2007; Nguyen et al., 2008 \\
\hline $\begin{array}{l}\text { Studies suggesting that microvascular abnormality is only relevant in } \\
\text { some subtypes of depression, for example, depression due to a general } \\
\text { medical condition like atherosclerosis, myocardial ischemia, } \\
\text { hypertension, diabetes mellitus or inflammation }\end{array}$ & $\begin{array}{l}\text { Thomas et al., 2001; Santos et al., 2009; Vaccarino et al., 2009; Nguyen et al., 2010; } \\
\text { Hajjar et al., 2011; Kim et al., 2011; Santos et al., 2012; Wolfson et al., 2013; Tchalla et } \\
\text { al., 2015; Gorska-Ciebiada et al., } 2015\end{array}$ \\
\hline $\begin{array}{l}\text { Studies assuming that microvascular abnormality is only relevant in } \\
\text { late-life depression }\end{array}$ & $\begin{array}{l}\text { Ballard et al., 2000; Thomas et al., 2001; Patankar et al., 2007; Santos et al., 2009; } \\
\text { Hajjar et al., 2011; Kim et al., 2011; Sinka et al., 2012; Wolfson et al., 2013; Tchalla et al., } \\
\text { 2015; Gorska-Ciebiada et al., } 2015\end{array}$ \\
\hline $\begin{array}{l}\text { Study presuming that microvascular abnormality is only significant in } \\
\text { pharmacoresistant depression }\end{array}$ & Patankar et al., 2007 \\
\hline
\end{tabular}

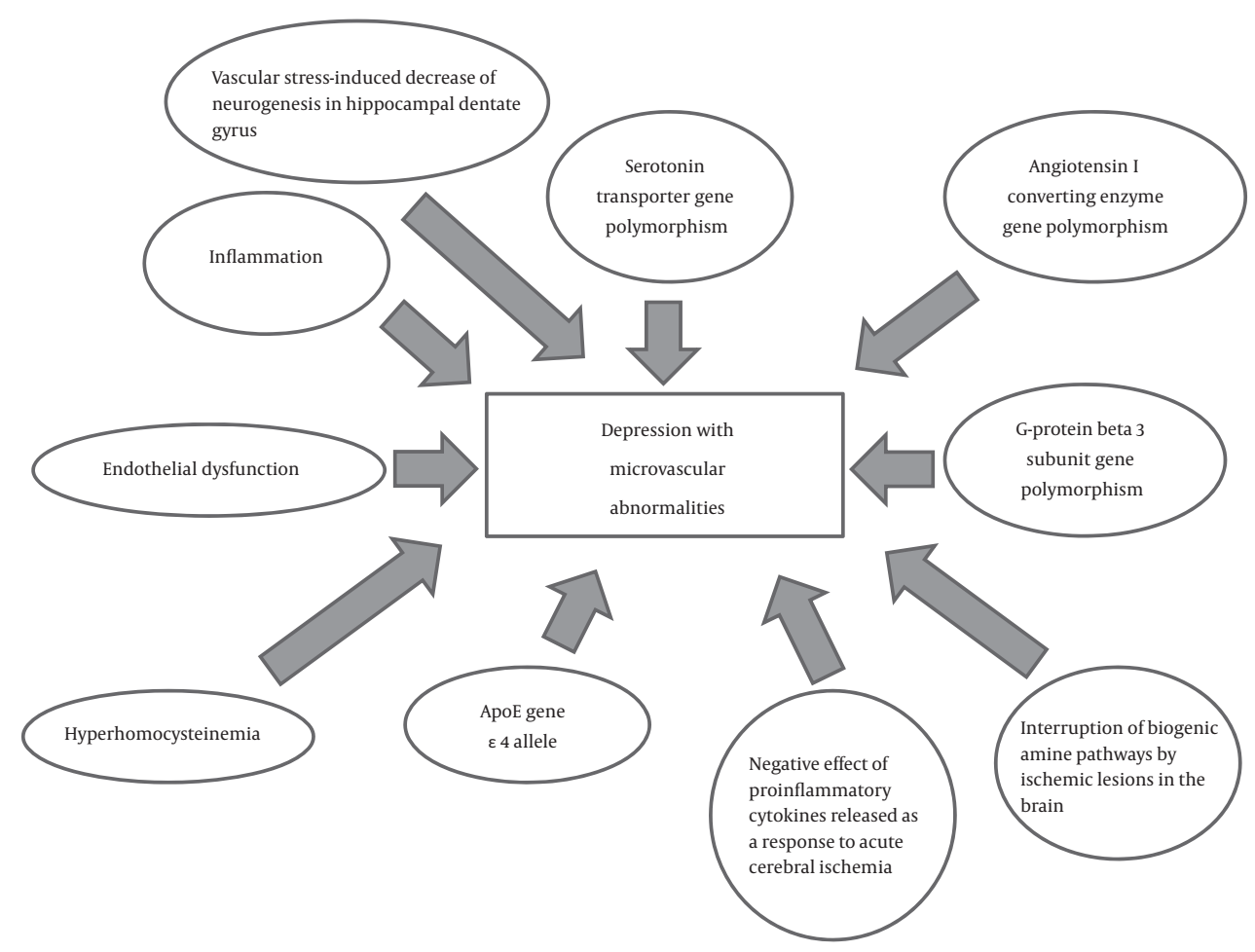

Figure 1. Biological Mechanisms of Depression With Microvascular Abnormalities

that a vascular stress-induced decrease in the neurogenesis of the hippocampal dentate gyrus may bring on depressive episodes $(9,29)$. Disturbed adult neurogenesis, possibly leading to a malfunctioning hippocampus, may add to the cognitive deficits and reduced hippocampal volumes seen in depressed patients.

Several avenues for further research into microvascular pathology in clinical depression, which may also include the assessment of retinal vessels, are possible:
- Whole-genome association studies identifying the genes responsible for microvascular changes, depression and their overlap, including their epigenetic status in various ethnic groups

-A more intense evaluation of the role of inflammation biomarkers in the development of depression

- More precise identification of depression subtypes in which microvascular abnormality is significant

- Exploration of unipolar, bipolar and adjustment de- 
pression separately as of a possible microvascular pathology

- Research aimed at finding specific brain loci in which microvascular disorders may trigger depression

- Looking for psychosocial variables important in the development of the microvascular subtype of depression

- Gene-Environment Wide Interaction Studies (GEWIS) in which multiple gene vs gene, gene vs environment and environment vs environment interactions are evaluated simultaneously, taking the statistical problem of multiple comparisons into account

- Longitudinal studies in depressed patients focused on their microvascular pathology and a possible relationship to the clinical state

- Psychiatric and ophthalmological examination of offspring of the patients with depression and microvascular pathology

- Psychopharmacological studies of antidepressants and other medications (e.g. anti-inflammatory drugs) efficient in the treatment of depression accompanied by microvascular abnormality

From the clinical point of view, it seems to be reasonable to actively search for physical illnesses, which contribute to depression associated with a microvascular pathology, (e.g. hypertension, diabetes, and inflammation) in depressed patients, and to treat them sufficiently.

\section{Conclusion}

Most studies support the idea that at least some subtypes of depression are associated with microvascular abnormality. Further research should be aimed at identifying the genetic background of depression accompanied by microvascular changes, searching for other biological mechanisms common in depression and microvascular pathology, longitudinal studies of this subcategory of depressed patients, and possible pharmacotherapy overcoming the usual non-responsiveness. Last but not least, an active detection and aggressive treatment of physical diseases like hypertension or diabetes associated with microvascular pathology and depression is warranted (Corriere et al., 2013; Khavandi et al., 2013) $(30,31)$.

\section{Footnotes}

Authors' Contribution: Evgenii Sadykov, Ladislav Hosak and Jan Studnicka conceived and designed the evaluation and did the literature search and review. Evgenii Sadykov, Ladislav Hosak, Jan Studnicka, Xavier Fung, Khurum Hakeem, Ashwin Mangarai, Ishleen Kaur, Gloria Mbuebue, Stefanos Kateroglou, and Mikela-Rafaella Siligardou interpreted and analyzed the data. Evgenii Sadykov, Ladislav
Hosak, Jan Studnicka, Xavier Fung, and Khurum Hakeem revised the manuscript critically for important intellectual content. Ladislav Hosak supervised the study. Xavier Fung was the native English speaker, who corrected the draft of the manuscript. All authors read and approved the final manuscript.

Declaration of interest: The authors declare no conflict of interest related to this article.

Financial Disclosure: The authors had no financial interests related to the material in the manuscript.

Funding/Support: The work was supported by the grant SVV-2015-260178 by Charles university in Prague, Czech Republic, and by the grant AZV MZ CR 16-27243A by the ministry of health, Czech Republic.

Role of the Sponsor: The sponsors of the study were Charles university in Prague, Czech Republic, and the ministry of health, Czech Republic. These public institutions had no role in the design and conduct of this review and approval of the manuscript.

\section{References}

1. World Health Organization. . Depression a global public health concern 2015. Available from: http://www.who.int/mental_health/ management/depression/who_paper_depression_wfmh_2012.pdf? $\mathrm{ua}=1$.

2. World Health Organization . Women's health 2015. Available from: http://www.who.int/topics/womens_health/en.

3. The World Bank. . Mental health 2015. Available from: http://www. worldbank.org/en/topic/health/brief/mental-health.

4. Sturm R, Wells KB. How can care for depression become more costeffective? JAMA. 1995;273(1):51-8. [PubMed: 7996651].

5. Camus V, Kraehenbuhl H, Preisig M, Bula CJ, Waeber G. Geriatric depression and vascular diseases: what are the links? J Affect Disord. 2004;81(1):1-16. doi: 10.1016/j.jad.2003.08.003. [PubMed: 15183594].

6. Santos M, Kovari E, Hof PR, Gold G, Bouras C, Giannakopoulos P. The impact of vascular burden on late-life depression. Brain Res Rev. 2009;62(1):19-32. doi: 10.1016/j.brainresrev.2009.08.003. [PubMed: 19744522].

7. Gordon MS. The synaptic organization of the brain. 5 ed. Oxford University Press; 2004.

8. Hosak L, Hakeem K, Raad M, Studnicka J. Is microvascular abnormality a new endophenotype in schizophrenia? Psychiatr Danub. 2015;27(3):225-9. [PubMed: 26400129].

9. Santos M, Gold G, Kovari E, Herrmann FR, Bozikas VP, Bouras C, et al. Differential impact of lacunes and microvascular lesions on poststroke depression. Stroke. 2009;40(11):3557-62. doi: 10.1161/STROKEAHA.109.548545. [PubMed: 19696424].

10. Santos M, Gold G, Kovari E, Herrmann FR, Hof PR, Bouras C, et al. Neuropathological analysis of lacunes and microvascular lesions in lateonset depression. Neuropathol Appl Neurobiol. 2010;36(7):661-72. doi: 10.1111/j.1365-2990.2010.01101.x. [PubMed: 20609111].

11. Ballard C, McKeith I, O’Brien J, Kalaria R, Jaros E, Ince P, et al. Neuropathological substrates of dementia and depression in vascular dementia, with a particular focus on cases with small infarct volumes. Dement Geriatr Cogn Disord. 2000;11(2):59-65. [PubMed: 10705161].

12. Thomas AJ, Ferrier IN, Kalaria RN, Perry RH, Brown A, O'Brien JT. A neuropathological study of vascular factors in late-life depression. J Neurol Neurosurg Psychiatry. 2001;70(1):83-7. [PubMed: 11118253]. 
13. Sinka L, Kovari E, Santos M, Herrmann FR, Gold G, Hof PR, et al. Microvascular changes in late-life schizophrenia and mood disorders: stereological assessment of capillary diameters in anterior cingulate cortex. Neuropathol Appl Neurobiol. 2012;38(7):696-709. doi: 10.1111/j.1365-2990.2012.01263.x. [PubMed: 22360590].

14. Hajjar I, Quach L, Yang F, Chaves PH, Newman AB, Mukamal K, et al. Hypertension, white matter hyperintensities, and concurrent impairments in mobility, cognition, and mood: the Cardiovascular Health Study. Circulation. 2011;123(8):858-65. doi: 10.1161/CIRCULATIONAHA.110.978114. [PubMed: 21321150].

15. Wolfson L, Wakefield DB, Moscufo N, Kaplan RF, Hall CB, Schmidt JA, et al. Rapid buildup of brain white matter hyperintensities over 4 years linked to ambulatory blood pressure, mobility, cognition, and depression in old persons. J Gerontol A Biol Sci Med Sci. 2013;68(11):138794. doi: 10.1093/gerona/glt072. [PubMed: 23766429].

16. Patankar TF, Baldwin R, Mitra D, Jeffries S, Sutcliffe C, Burns A, et al. Virchow-Robin space dilatation may predict resistance to antidepressant monotherapy in elderly patients with depression. J Affect Disord. 2007;97(1-3):265-70. doi: 10.1016/j.jad.2006.06.024. [PubMed: 16919335].

17. Vaccarino V, Votaw J, Faber T, Veledar E, Murrah NV, Jones LR, et al. Major depression and coronary flow reserve detected by positron emission tomography. Arch Intern Med. 2009;169(18):1668-76. doi: 10.1001/archinternmed.2009.330. [PubMed: 19822823].

18. Cheung N, Rogers S, Mosley TH, Klein R, Couper D, Wong TY. Vital exhaustion and retinal microvascular changes in cardiovascular disease: atherosclerosis risk in communities study. Psychosom Med. 2009;71(3):308-12. doi: 10.1097/PSY.0b013e318190fo09. [PubMed: 19073748].

19. Jensen RA, Shea S, Ranjit N, Diez-Roux A, Wong TY, Klein R, et al. Psychosocial risk factors and retinal microvascular signs: the multiethnic study of atherosclerosis. Am JEpidemiol. 2010;171(5):522-31. doi: 10.1093/aje/kwp414. [PubMed: 20035010].

20. Ikram MK, Luijendijk HJ, Hofman A, de Jong PT, Breteler MM, Vingerling JR, et al. Retinal vascular calibers and risk of late-life depression: The Rotterdam Study. Am J Geriatr Psychiatry. 2010;18(5):452-5. [PubMed: 20429085]

21. Sun C, Tikellis G, Klein R, Steffens DC, Larsen EK, Siscovick DS, et al. Are microvascular abnormalities in the retina associated with depression symptoms? The Cardiovascular Health Study. Am J Geriatr Psychiatry. 2007;15(4):335-43. doi: 10.1097/01.JGP.0000247161.98311.0f. [PubMed: 17384316]
22. Kim DH, Newman AB, Hajjar I, Strotmeyer ES, Klein R, Newton E, et al. Retinal microvascular signs and functional loss in older persons: the cardiovascular health study. Stroke. 2011;42(6):1589-95. doi 10.1161/STROKEAHA.110.605261. [PubMed: 21493913].

23. Li LJ, Ikram MK, Broekman L, Cheung CY, Chen H, Gooley JJ, et al. Antenatal Mental Health and Retinal Vascular Caliber in Pregnant Women. Transl Vis Sci Technol. 2013;2(2):2. doi: 10.1167/tvst.2.2.2. [PubMed: 24049713].

24. Meier MH, Gillespie NA, Hansell NK, Hewitt AW, Hickie IB, Lu $\mathrm{Y}$, et al. Associations between depression and anxiety symptoms and retinal vessel caliber in adolescents and young adults. Psychosom Med. 2014;76(9):732-8. doi: 10.1097/PSY.0000000000000117. [PubMed: 25373892].

25. Nguyen TT, Wong TY, Islam FM, Hubbard L, Ajilore O, Haroon E, et al. Evidence of early retinal microvascular changes in patients with type 2 diabetes and depression. Psychosom Med. 2010;72(6):535-8. doi: 10.1097/PSY.ob013e3181da90f4. [PubMed: 20368470].

26. Nguyen TT, Wong TY, Islam FM, Hubbard L, Miller J, Haroon E, et al. Is depression associated with microvascular disease in patients with type 2 diabetes? Depress Anxiety. 2008;25(11):E158-62. doi: 10.1002/da.20427. [PubMed: 17966124].

27. Tchalla AE, Wellenius GA, Sorond FA, Travison TG, Dantoine T, Lipsitz LA. Elevated circulating vascular cell Adhesion Molecule-1(sVCAM-1) is associated with concurrent depressive symptoms and cerebral white matter Hyperintensities in older adults. BMC Geriatr. 2015;15:62. doi: 10.1186/s12877-015-0063-7. [PubMed: 26040277].

28. Gorska-Ciebiada M, Saryusz-Wolska M, Borkowska A, Ciebiada M Loba J. Serum levels of inflammatory markers in depressed elderly patients with diabetes and mild cognitive impairment. PLoS One. 2015;10(3):0120433. doi: 10.1371/journal.pone.0120433. [PubMed: 25793613].

29. Santos M, Xekardaki A, Kovari E, Gold G, Bouras C, Giannakopoulos P. Microvascular pathology in late-life depression. J Neurol Sci. 2012;322(1-2):46-9. doi: 10.1016/j.jns.2012.05.048. [PubMed: 22687957].

30. Corriere M, Rooparinesingh N, Kalyani RR. Epidemiology of diabetes and diabetes complications in the elderly: an emerging public health burden. Curr Diab Rep. 2013;13(6):805-13. doi: 10.1007/s11892-013-04255. [PubMed: 24018732].

31. Khavandi K, Arunakirinathan M, Greenstein AS, Heagerty AM. Retinal arterial hypertrophy: the new LVH? Curr Hypertens Rep 2013;15(3):244-52. doi: 10.1007/s11906-013-0347-2. [PubMed: 23575736]. 